\title{
Game Theory based Incentive Mechanisms in Delay Tolerant Network
}

\author{
Shailesh P. Hulke \\ Research Scholar \\ Department of Computer and IT \\ College of Engineering, Pune
}

\author{
Vahida Attar, Ph.D. \\ Associate Professor \\ Department of Computer and IT \\ College of Engineering, Pune
}

\begin{abstract}
Delay Tolerant Networking is the art and science of moving bits around in bad situations, such as between distant endpoints in deep space, or among unknown mobile users at uncertain positions. It is an opportunistic network where there is lack of end to end connectivity between source and destination, so that the communications take place using store-carry-and-forward manner. The rational parties send the bundle to its destination using the basic store-carry-andforward method. DTN has a potential assumption that the nodes are willing to help other node for packet forwarding. But in reality, selfish behavior always exists. Many incentive schemes are proposed to address the selfish behavior of node. This paper analyzes a game theory based incentive mechanism to address the selfishness problem of node and stimulate the intermediate nodes to forward the bundles towards the destination node for better packet delivery.
\end{abstract}

\section{Keywords}

DTN, Store-Carry-and-Forward, Selfishness, Game Theory Based Incentives Mechanism.

\section{INTRODUCTION}

A Delay Tolerant Network (DTN) is a network of regional networks. It resides on top of regional networks and Internet is also part of it. DTN support interoperability of the networks which covers it by including long delays between and within regional networks. Many networks including Internet also may not have assumptions which Delay Tolerant network has. Characteristic of Delay Tolerant Networks are explained below:

Intermittent Connectivity: In a situation that, if there is no end to end connectivity present, then a network may divide into one or many parts called Network Partitioning and in such situation TCP/IP protocols does not work. Hence, protocols other than TCP/IP are required.

Long or Variable Delay: Apart from intermittent connectivity, variable queuing delays at nodes and long propagation delays between nodes contribute to end to end delays that may beat various applications and Internet protocols which depends on quick return of acknowledgments or data.

Asymmetric Data Rates: Users with cable TV which uses Internet supports moderate asymmetric nature of full duplex links which has bi-directional data rate. But if asymmetric nature is large, they can beat the conversational protocols.

High Error Rates: As the links are highly asymmetric, bit errors on links must be identified and corrected (which requires extra parity bits and more processing power of CPU) or retransmission of the sent packet (which causes more network traffic). Sometimes, retransmission of entire packets are needed including hop to hop rather than end to end retransmission.[1]

\subsection{Store and Forward Message Switching}

The problem associated with Delay tolerant network can be overcome using the store-and forward message switching. Store and forward message switching method is an old and ancient method like postal system. Entire message or fragment of application data are forwarded from a storage place on one site to another node which has storage capacity, followed by a path which reaches to the destination. For example, today's voice mail and e-mail systems are star relays having centralized monitoring where each device is connected to central storage device.[8]

The storage places like hard disk stores messages for long time, hence, it is called as persistence storage. The memory chips have a characteristic of storing a data for a very short time. Routers in the network store the packets or queue the packets for a very short time may be for milliseconds when they are looking up their routing table and outgoing router port.

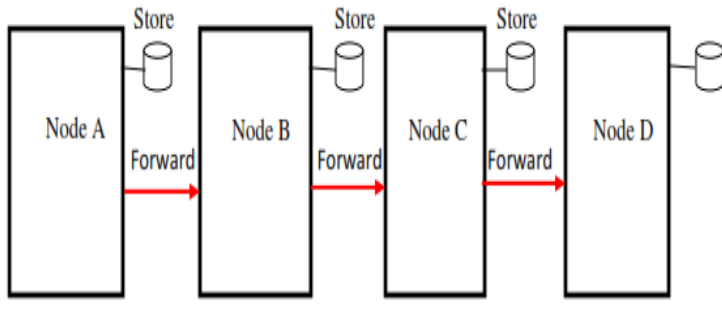

Figure 1. Store-carry-and-forward Mechanism

Routers that are used in the Delay Tolerant Network need persistent storage for their queuing of data packets for a long time for the reasons explained below [1]

Due to opportunistic characteristics of DTN, communication link between the nodes may not be available for a long time. Sending and receiving of data by one node may be faster or reliable than other node in the communicating node pair. Message retransmission is needed which is transmitted before any error occurs at an upstream node or link which is towards the destination, or if the upstream node decided to reject the forwarded packets. For single transfer of entire packet or the fragments of packet, the message switching technique is used which provides knowledge of the size of messages by the nodes of the network. Therefore, intermediate storage space and retransmission bandwidth is very essential in such type of networks. 


\subsection{DTN Protocol Stack}

The DTN architecture implements store-and-forward message switching by overlaying a new protocol layer called the bundle layer on top of heterogeneous region specific lower layers.[10] In heterogeneous network, TCP/IP stack is modified and one extra layer is added in between the transport layer and application layer. By using the store and forward mechanism, a persistent storage is obtained. Bundle layer is also used to achieve the interoperability among heterogeneous network.[11] The message in bundle layer is called as bundle, which consists of bundle header, control information and application data unit (ADU).

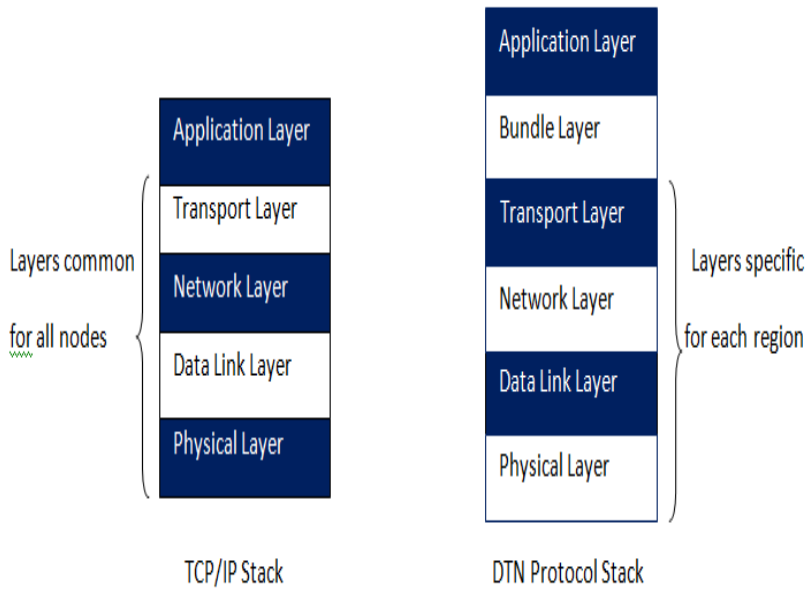

Figure 2: DTN specific protocol stack

\subsection{Selfish Behavior of DTN Nodes}

In Delay Tolerant Network, due to limited storage and battery constraints, nodes become selfish. Because of this selfish behavior of the node, it may be possible that packets or bundles are intentionally dropped even though a node has capability to forward the data. The selfish node try to maximize their own benefit by enjoying the services provided by the DTN while refusing to forward the bundles for others, this is known as routing misbehavior of the DTN. The three main issues of research interests in the aspect of cooperative techniques are:[9]
i. Seek for the non-cooperative nodes.
ii. The affections cooperation put on the performance of the whole network.
iii. Design protocols for the nodes' cooperation.

\subsection{An Overview of Game Theory}

Game Theory provides analytical tools to predict the outcome of complex interactions among rational entities. In a game, each individual player participates with a payoff depending upon the other players. In wireless ad-hoc network, player acts as a node in the networks, an action acts as a move, strategy acts as a plan of action during a game, and payoff is a reward given to the player for its action which is positive or negative.[7]

Game theory based Incentives mechanism is evolved to motivate and stimulate intermediate node to cooperate other intermediate node in order to do a successful delivery of bundles to its recipient.

\section{APPLICATION OF GAME THEORY TO AD HOC NETWORKS}

The game theory can be applied to get the following benefits:

Distributed systems: Game theory is a strong tool for analysis of distributed protocol which investigates the existence, uniqueness and convergence of nodes when they perform independent adaptations.

Cross layer optimization: In an ad-hoc network, the objective of game theory is to optimize the performance of layers while making some node decision at particular layer. For cross layer optimization, game theoretic analysis provides an insight approach by giving an appropriate formulation for the action space.

Design of incentive schemes: Incentive schemes are designed to detect and punish the selfish behavior of node using game theory.

\subsection{Game theory based Incentive Mechanisms}

This section tries to discuss various game theory based incentive mechanisms.

\subsubsection{Multicent Mechanism}

In Delay tolerant network, Communication opportunities are very limited, because of which packet forwarding affects its performance. Therefore, a multifunction Incentive scheme, i.e., Multicent is adapted to recognize the cooperation among the network in a given DTN networks using the game theory incentives system.[2] Multicent incentive scheme not only cooperates the node to send the packet but also encourages the node to follow defined rules to realize the desired performance objectives. Multicent also provides the Quality of service of packet routing for source, destination or SourceDestination pairs. A multicent incentive scheme has a payoff function that achieves the two aspect of cooperation:

The First Aspect of Cooperation (Forwarding Packets):-

In this aspect, while forwarding the packet, each packet is recognized either as it will reach to the destination or it fails. In multi-copy routing, packets are replicated rather than transferred to another node. Then, forwarding a packet does not affect the current nodes future opportunity to earn credits from this packet, since it still keeps the packet. The additional credit encourages the node to deliver packets to their destinations upon encountering them. Therefore, in order to maximize its profit, a node will be cooperative at every opportunity to forward a packet to other nodes or its destination.

The Second Aspect of Cooperation (Realizing Performance Objectives):-

Multicent actually can motivate nodes to realize any performance objective with a defined utility function, including the strategy to realize equal forwarding opportunity among packets

\subsubsection{GTFT Mechanism}

GTFT, a game theory based incentive system for DTN proposed by Vikram Srinivasan et al.[3]

As per the behavior of DTN network, the nodes have limited resources and battery constrains, as a result, the energy of the nodes is limited. Due to this energy constraints, the selfish behavior of the nodes occur in DTN, say, the nodes may not be willing to forward the packets to other. So the assumption 
that all the nodes are cooperative is unrealistic. The traffic generated by rational behavior of user will not always be willing to expand their energy resources. To resolve this problem, researchers had devised a scalable strategy which uses game theory approach known as GTFT algorithm. GTFT is also known as scalable acceptance algorithm. The node uses acceptance algorithm to decide whether a relay request should be accepted or rejected based on its past history. The GTFT algorithm is not a stationary strategy and has property to prevent the node from being exploited. It takes action which is solely based on locally gathered information.

\subsubsection{NDI Mechanism}

NDI, a game theory based incentive system for DTN proposed by Ruiyun Yu at el.[4]

In DTN Network, there is no end to end connectivity or path between the source and destination to forward the bundles in opportunistic environment, so the communication takes place by the store-carry-forward strategy. Selfish behavior of node purposely drops the packet in the route, so the performance of the whole network is degraded. Incentive mechanism is the best solution to find and punish the selfish node in order to forward the bundles which improves the reliability and robustness of the networks. In this paper, researcher proposed the Node-dependence-based Dynamic gaming Incentive (NDI) algorithm, in which the nodes are motivated to relay packets from one node to another by exploiting the dynamic repeated gaming. The NDI algorithm presents a mechanism to resolve the selfish behavior of nodes. The methods are being designed for reward and punishment which are based on the node dependence degree.

\subsubsection{GA Mechanism}

GA, a game theory based incentive system for DTN proposed by Marcin Seredynski et al.[5]

In this paper, researcher addresses the problem of the selfish behavior in self-policing ad-hoc networks. To resolve the problem of selfish behavior of node in ad-hoc network, researcher proposed a new game theory based model of the network namely Genetic Algorithm (GA).

The GA also does cooperation between the node which takes place in two manner as follows:

i. Firstly, it assumes that only two players participate in each game.

ii. Secondly, it models a specific dilemma situation described by the Prisoner Dilemma payoff Table.

\subsubsection{PFG Mechanism}

PFG, a game theory based incentive system for DTN proposed by Lei Yin et al.[6]

Table 1 A Comparison of Game Theory based incentive mechanisms

\begin{tabular}{|l|l|l|l|l|}
\hline $\begin{array}{l}\text { Sr. } \\
\text { No. }\end{array}$ & $\begin{array}{l}\text { Game Theory } \\
\text { Based Incentives } \\
\text { Mechanism }\end{array}$ & Basic Idea & Advantages & Disadvantages \\
\hline 1 & Multicent & $\begin{array}{l}\text { Takes full advantages of forwarding } \\
\text { opportunities and gives higher priorities to } \\
\text { the packets or bundles }\end{array}$ & $\begin{array}{l}\text { Greater Improvement to } \\
\text { hit rate in forwarding and } \\
\text { storing }\end{array}$ & $\begin{array}{l}\text { It cannot motivate } \\
\text { nodes to forward } \\
\text { packets in different } \\
\text { sequence to achieve } \\
\text { different performance } \\
\text { objectives. }\end{array}$ \\
\hline
\end{tabular}

In Delay tolerant network, node's behavior is selfish due to the constraints of buffer and energy which does not cooperate in forwarding bundles. In this paper, researcher proposed a game theory based a pay-for-gain (PFG) algorithm, to investigate equilibrium condition which uses loan-credit theory to motivate and maximize each nodes interest. Incentive scheme's effectiveness is evaluated by the researcher using the traces of DTN from a part of Helsinki. To ensure the cooperation among the selfish nodes, PFG uses variable initial shared buffer space which increases the total delivered traffic to 70 percent as full cooperation.

The main contribution of this research can be summarized as follow

i. For optimizing the benefits without degradation of the network performance, an incentives mechanism need to be develop which is based on game theory and loan credit.

ii. Using PFG, each nodes's interest is maximized by the investigate equilibrium condition.

iii. Effectiveness of incentives schemes is evaluated by the researcher using the traces of DTN from a part of Helsinki.

\section{COMPARISON OF INCENTIVE MECHANISMS}

Section 2 analyzes different Game Theory based incentive mechanisms and this section presents a comparative study. The basic idea, advantage and disadvantage of each strategy are qualitatively listed. Table 1 shows a comparison of Game Theory based incentive mechanisms.

From the comparison, following understandings can be drawn:

i. In Game theory based incentives mechanism, each individual player participated whose payoff depends on the other player.

ii. Limited energy (i.e. battery power) and limited buffer space of DTN nodes are the main reasons for the selfish behavior of DTN nodes.

The game-theory based incentive mechanism performs well when the number of nodes is few. But, in real scenario, the number of nodes might be large and it may not perform well.[9] Therefore, there is a need to develop a strategy or mechanism in order to work well in a network of large selfish nodes in DTN which demands more research work in future. 


\begin{tabular}{|c|c|c|}
\hline 2 & GTFT & $\begin{array}{l}\text { A player adopts an aspects of cooperation } \\
\text { as long as the other side does }\end{array}$ \\
\hline 3 & NDI & $\begin{array}{l}\text { Based on the Node Dependence } \\
\text { Degree(NDD) } \\
\text { Tolerance Mechanism }\end{array}$ \\
\hline 4 & GA & Strategy based on the trust level \\
\hline 5 & PFG & $\begin{array}{l}\text { Finite Population of node in network } \\
\text { Network operate in discrete time }\end{array}$ \\
\hline
\end{tabular}

\section{CONCLUSION}

This paper discusses different Game Theory based Incentive Mechanisms and analyze them on their advantages and disadvantages. These incentive mechanisms stimulate the nodes for packet forwarding along with detection of selfish nodes. Game theory based incentive mechanisms are good enough to enforce high level of cooperation among the nodes and are interested in sending their own packets. DTN still demands more research for selfish behavior of nodes so that selfish nodes also should take part in delivering the packets to the destination. Security of DTN nodes and Energy of DTN nodes are the two major areas in DTN which demands more research work in future.

\section{REFERENCES}

[1] F. Warthman, Delay Tolerant Network, A Tutorial March 2003. http://www.dtnrg.org/docs/tutorials/warthman-1.1.pdf

[2] Kang Chen and Haiying Shen, "Multicent: A Multifunctional Incentive Scheme Adaptive to Diverse Performance Objectives for DTN Routing", IEEE International Conference on Sensing, Communications and Networking (SECON),2013.

[3] Vikram Srinivasan, Pavan Nuggehalli, Carla-Fabiana Chiasserini, and Ramesh R. Rao, "An Analytical Approach to the Study of Cooperation in Wireless Ad Hoc Networks",IEEE TRANSACTIONS ON WIRELESS COMMUNICATIONS, VOL. 4, NO. 2, MARCH 2005.

[4] Ruiyun Yu Pengfei Wang Zhijie Zhao, "NDI:Nodedependence-based Dynamic gaming Incentive algorithm

\begin{tabular}{|l|l|}
$\begin{array}{l}\text { Address issues of traffic } \\
\text { relaying } \\
\text { Distributed and Scalable }\end{array}$ & $\begin{array}{l}\text { Fail to guarantee proper } \\
\text { level of cooperation }\end{array}$ \\
\hline $\begin{array}{l}\text { Effective on increase the } \\
\text { delivery ratio and decrease } \\
\text { average latency }\end{array}$ & $\begin{array}{l}\text { Effective only when lot } \\
\text { of selfish node in the } \\
\text { network }\end{array}$ \\
\hline $\begin{array}{l}\text { Maximize the throughput } \\
\text { of the network by } \\
\text { enforcing cooperation }\end{array}$ & $\begin{array}{l}\text { False accusations, } \\
\text { temporary failure, noise }\end{array}$ \\
$\begin{array}{l}\text { More effective in } \\
\text { stimulating cooperation } \\
\text { among selfish node }\end{array}$ & $\begin{array}{l}\text { It assumes topology of } \\
\text { network doesn't change } \\
\text { and communication } \\
\text { between nodes can't be } \\
\text { disconnected }\end{array}$ \\
\hline
\end{tabular}

in opportunistic networks", National Natural Science Foundation of China under Grant No. 61272529; the Fundamental Research Funds for the Central Universities under Grant No.N120417002.

[5] Marcin Seredynski Pascal Bouvry Mieczyslaw A. Klopotek, "Modelling the Evolution of Cooperative Behavior in Ad Hoc Networks using a Game Based Model", Proceedings of the 2007 IEEE Symposium on Computational Intelligence and Games (CIG 2007).

[6] Lei Yin, Hui-mei Lu, Yuan-da Cao, Jian-min Gao, "Cooperation in Delay Tolerant Networks", 2010 2nd International Conference on Signal Processing Systems (ICSPS).

[7] Vivek Srivastava, James Neel, Allen B. MacKenzie, Rekha Menon, Luiz A. DaSilva, James E. Hicks, Jeffrey H. Reed, Robert P. Gilles, "Using Game Theory to Analyze Wireless Ad Hoc Networks".

[8] Upendra Malekar, Lalit Kulkarni, "Analyzing Credit Based Incentive Mechanisms in Delay Tolerant Network: A Survey" International Journal of Advance Foundation and Research in Computer (IJAFRC), Volume 1, Issue 12, December 2014. ISSN 23484853.

[9] Xin Jiang, Xiang-Yu Bai, "A Survey on Incentive Mechanism of Delay Tolerant Networks", 2013 IEEE.

[10] K. Scott, S. Burleigh, Bundle protocol specification, http://www.rfceditor.org/rfc/pdfrfc/rfc5050.txt.pdf

[11] S. Symington, S. Farrell, H. Weiss, P. Lovell, Bundle security protocol specification, http://www.rfceditor.org/rfc/pdfrfc/rfc6257.txt.pdf 